Apidologie, 1974, 5 (2), 127-147.

\title{
ZUR FRAGE DES KONIGINNENGEWICHTES IN ABHÄNGIGKEIT VON UMLARVALTER], UND LARVENVERSORGUNG
}

\author{
Sur la question du poids des reines \\ en rapport avec l'âge de la larve transférêe et l'alimentation \ẩdes larves
}

Karl WEISS

Bayerische Landesanstalt für Bienenzucht, Erlangen

\section{SUMMARY}

\author{
THE WEIGHT OF HONEYBEE QUEENS SEEN IN DEPENDANCE \\ OF THE LARVA'S GRAFTIYG AGE AND ITS FOOD SUPPLY
}

Honeybee queens were reared from newly hatched until almost 48 hours old larvae of the same descent and in the same nurse colony. A comparison of pupal weights did not show secure differences within the various breedings. By a parameter free test method, a comparison of all relevant breedings, only, allowed to prove an slight advantage in weight of those queens raised from newly emerged larvae compared with those reared from $24 \mathrm{~h}$ old larvae and almost $48 \mathrm{~h}$ old ones. Queen pupae reared from larvae slightly older than $48 \mathrm{~h}$ were significantly lighter in weight than those raised from $24 \mathrm{~h}$ old larvae and younger ones.

Larvae grafted when $48 \mathrm{~h}$ old, after 24 hours, disposed of more brood food than those grafted 24. h old, these having more provisions than newly emerged ones. Nevertheless after pupation the food remainders in the queen cells decreased gradually, corresponding to the larval age at grafting time. The quantity of remaining food is appointed by the total royal nursing times of a larva. It is also reflected by the length the queen cell has reached.

Whilst the quantity of remaining food in several queen breedings, practiced successively in queenless nurse colonies, from the start on, rapidly decreased to zero, the weights of the queens in the first breeding series were nearly distributed at random.

Under the same presupposition, as for the larval age and the size of queen cell cups, and when queens are raised at the same time in the same nurse colony there exists no dependence between the quantity of food remainders in the queen cells and the weights of pupae.

An attempt is made to explain the apparent contradiction of worker bee larvae growing faster than queen larvae during the first three larval days and on the other hand worker bee larvae grafted when older than $48 \mathrm{~h}$ developing into smaller queens then those of younger larval stages.

In practical queen breeding a larval grafting age unto $36 \mathrm{~h}$ can be pleaded for, as the differences in weight of queens developing from larvae grafted in the time between zero and almost $48 \mathrm{~h}$ are practically insignificant. 


\section{ZUSAMMENFASSUNG}

Beim Vergleich von Königinnen, die aus eben geschlüpften bis knapp 2-tägigen Maden gleicher Abstammung zur gleichen Zeit im gleichen Pflegevolk gezüchtet wurden, ließen sich innerhalb der einzelnen Zuchten keine gesicherten Unterschiede der Puppengewichte festtellen. Erst bei Zusammenfassung aller einschlägigen Zuchten konnte mit einem prarameterfreien Testverfahren ein geringer Gewichtsvorteil der Königinnen aus eben geschlüpften Maden gegenüber solchen aus 1- und knapp 2-tägigen Larven nachgewiesen werden. Dagegen entwickelten sich aus Maden, die nur wenig über 2 Tage alt waren, deutlich leichtere Königinnenpuppen als aus l-tägigen und jüngeren Tieren.

Zweitägig umgelarvte Maden verfügten nach 24 Stunden über mehr Futtersaft als 1 tägig umgebettete, und diese wiederum waren besser versorgt als eben geschlüpfte. Dennoch nahmen die nach der Verpuppung in den Weiselzellen zurückbleibenden Restfuttermengen beim Umlarven von eben geschlüpften, 1- und 2-tägigen Maden stufenweise ab. Die Größe der Restfuttermenge wird durch die Gesamtzeit der königlichen Pflege einer Made bestimmt. Sie spiegelt sich außerden in der erreichten Länge der Weiselzelle wider.

Während die Restfuttermengen bei mehreren hintereinander in entweiselten Pflegevölkern durchgeführten Zuchten von Anfang an sehr rasch bis nahe Null abnahmen, waren die Königinnengewichte in den ersten Zuchtserien annähernd zufällig verteilt.

Unter gleichen Voraussetzungen bezüglich Larvenalter und Größe der Weiselbecher und bei Aufzucht zur gleichen Zeit im gleichen Pflegevolk besteht keine Abhängigkeit zwischen den in den Weiselzellen zurückbleibenden Restfuttermengen und den Puppengewichten.

Es wird versucht, den scheinbaren Widerspruch zu erklären, daß Arbeitermaden während der erstent 3 Larventage schneller wachsen als Königinnenmaden, andererseits aber über 2 Tage alte Arbeitermaden kleinere Königinnen ergeben als jüngere Umlarvstadien.

Für die Züchterpraxis sind die schwer zu sichernden Gewichtsunterschiede zwischen Königinnen, die aus Larven verschiedenen Alters bis zu knapp 2 Tagen hervorgehen, so gering, daß ein Umlarvalter bis zu $11 / 2$ Tagen auch im Hinblick auf die Größenentwicklung der Zuchtköniginnen voll vertretbar erscheint.

In der künstlichen Nachschaffungszucht, welche nach weltweiter Gepflogenheit junge Maden als Aufzuchtmaterial verwendet, geht der königlichen Pflege eine mehr oder weniger lange arbeitermäßige Futterversorgung der Larven voraus. Während man einerseits weiß, daß schon auf relativ frühem Larvenalter Unterschiede in der Zusammensetzung des Futtersaftes von Arbeiterinnen- und Königinnenmaden bestehen und frühzeitige Wachstumsund Stoffwechselunterschiede zwischen den Larven beider Kasten auftreten, ließen sich anderseits bei der Verwendung von $1 / 2$ bis $1 \frac{1}{2}$-tägigen Arbeitermaden zur Königinnenaufzucht keinerlei Abweichungen in den Merkmalen der erwachsenen Tiere gegenüber den aus Eiern aufgezogenen nachweisen (WEIß, 1971; dort auch einschlägige Literatur). Fraglich blieb seinerzeit, ob nicht vielleicht erste echte Gewichtsunterschiede auftreten können.

Verschiedene Angaben in der Literatur scheinen hierfür zu sprechen. Wolosiewitsch (1954) und Nikolad (1969) fanden Schwarmköniginnen größer, als aus Larven gezüchtete Tiere. Weaver (1957), Jordan (1960) und Woyke (1967) stellten kontinuierlich abfallende Gewichtstendenzen bei Königinnen aus $0,1,2,3$, (und 4)-tägigen Maden fest. Nach Krasnopojew (1949) und Lawrechiv (1963) sollen aus dem. Ei gezüchtete Königinnen mehr gewogen haben, als solche aus jungen Larven. 
Solcherlei Mitteilungen veranlaßten mich, meine früheren Untersuchungen über die Abhängigkeit des Adultgewichtes der Königinnen vom Aufzuchtalter der Larven auf eine breitere Basis zu stellen. Außerdem war eine eigenartige Beobachtung von damals zu überprüfen, wonach beim Vergleich der Puppengewichte von Königinnen aus 1- und 2-tägigen Maden gleicher Abstammung die aus 1-tägigen Larven gezogenen Tiere in den zwei ersten von drei aufeinanderfolgenden Zuchtserien schwerer, in der dritten aber leichter waren als die Puppen aus 2 Tage alten Larven. Sollte das mehr als eine Zufallsbeobachtung gewesen sein, etwa derart, daß ältere $P$ flegevölker generell ältere Maden besser pflegen würden als jüngere?

Nebenher machte ich Beobachtungen über die Futterversorgung von Maden und Puppen und stellte die Frage nach einer möglichen Beziehung zwischen Futterversorgung und Königinnengewicht.

\section{I. - BESCHAFFUNG DES UNTERSUCHUNGSMATERIALS}

In der Regel sperrte ich die Königin des Pflegevolkes 9 Tage vor Zuchtbeginn über Absperrgitter in den Honigraum, um sie erst 2 Stunden vor dem ersten Zuchtansatz mit der offenen Brut aus dem Volk zu entfernen. Die Bienen wurden in einer Zarge mit ausschließlich gedeckelter Brut zusammengedrängt. Seltener entfernte ich die Königin und die Waben mit überwiegend offener Brut auch gleich aus dem Volk und begann ohne Verzug mit dem Züchten. Eine spätere Kontrolle auf wilde Nachschffungszellen war dann unumgänglich. Fünf Tage nach jedem Zuchtansatz - also kurz nach der Deckelung der Weiselzellen, manchmal auch im Turnus von 7 Tagen, wechselte ich den alten Zuchtrahmen durch einen neuen aus und brachte die gedeckelten Weiselzellen im Brutschrank bei $35^{\circ} \mathrm{C}$ und $60-70 \%$ r. F. unter. Die Pflegevölker erhielten während der Aufzucht kein Reizfutter, auch nicht bei Trachtmangel. Sie waren aber immer reichlich mit Honig und Pollen versorgt.

In den ersten Zuchten verwendete ich getauchte Weiselbecher aus Wachs von $9 \mathrm{~mm}$ innerer Randweite, später arbeitete ich ausschließlich mit Bechern aus Kunststoff der Firma Daniels et Co., Picayune, Mississippi. Die Bienen nahmen diese ebensogut an wie Wachsbecher. Die Weiselnäpfchen an den Zuchtlatten belarvte ich in abwechselnder Reihenfolge mit Maden verschiedenen Alters. Es wurde “ trocken » umgelarvt, d.h. ich beschickte die Näpfchen vorher nicht mit Futtersaft.

Die nach dem Alter datierten Maden erhielt ich durch Absperren der Zuchtkönigin unter einen Aufsteckrahmen aus Absperrgitter auf einer leeren Wabe. Der Rahmen bedeckte nur einen Teil der Wabenfläche. Meist nahm ich die Königin schon nach 6 Studen heraus, um sie später je nach Bedarf auch noch auf anderen Teilen der Zuchtwabe abzusperren. So standen mir nach angemessener Zeit verschiedene Altersstadien von Larven auf der gleichen Wabe zum Umlarven zur Verfügung.

Zwei Tage vor dem erwarteten Schlupf der Königinnen schnitt ich die Puppen aus den Zellen und ermittelte ihr Gewicht (vergl. WEIss 1967). Außerdem wog ich die zurückgebliebenen Futtersaftreste, die ich zu diesem Zweck mit einem Spatel aus den Zellen herausholte. Dieses Überschußfutter war zum Zeitpunkt der Wägung breiig, gummiartig oder völlig eingetrocknet. Da seine Konsistenz von der abgelagerten Futtermenge abhing, lie $B$ die Bestimmung des "Frischgewichtes " die bestehenden Unterschiede von Zelle zu Zelle und von Zuchtserie zu Zuchteserie nur umso deutlicher hervortreten.

Alle Versuche bis 1971 wurden mit Carnica-Bienen in Erlangen durchgeführt. Um die Variabilität der zu vergleichenden Königinnen so klein als möglich zu halten, verwendete ich 1971 zur Gewinnung des Zuchtstoffs eine Königin, die nach vorausgegangener Inzucht mit nur einem Geschwisterdrohn künstlich besamt worden war. Die Bereitstellung der Königin verdanke ich Prof. F. Ruttner in Oberursel. 1972 dienten zur Pflege und als Zuchtstofflieferanten Italiener-Völker. Diese Versuche führte ich während eines Studienaufenthaltes in den USA an der Universität von Georgia in Anthens durch. Dem Leiter der Entomologischen Abteilung Prof. H. Lund und dem Vorstand des Bienenlaboratoriums Prof. A. Dietz bin ich für ihre Hilfsbereitschaft und Unterstützung zu besonderem Dank verp flichtet. 


\section{II. - GEWICHTSVERGLEICH DER AUS VERSCHIEDEN ALTEM ZUCHTSTOFF AUFGEZOGENEN KONIGINNEN}

In Tabelle 1 sind alle gelungenen Zuchten, die mit verschieden altem Zuchtstoff in jeweils verschiedenen $P$ flegevölkern und $P$ flegefolgen durchgeführt wurden, mit Annahmezahlen, Gewichten, Gewichtsdifferenzen und statistischen Angaben wiedergegeben. Jede Zeile enthält das Ergebnis eines Zuchtansatzes.

Als Hinweis für das ungefähre Alter der Pflegebienen sind Pflegefolge und die Zeitdauer vom Entweiseln bis zum Zuchtansatz von Bedeutung (Spalte 2 u. 3). Die verschiedenen Pflegefolgen sind manchmal auseinandergerissen, um Unterschieden im Vergleichsalter der Maden besser gerecht zu werden. So sind die ältesten Maden in den Vergleichsstadien der Zuchten 1-21 (1. Versuchsgruppe) höchstens 2 Tage, die in den Zuchten 22-32 (2. Versuchsgruppe) jedoch über 2 Tage alt. Außerdem weisen die Pflegefolgen Lücken auf, die durch Verwendung der Pflegevölker zu Zuchten mit anderer Fragestellung verursacht sind.

Als überraschendes Ergebnis dieser Versuche stellt sich heraus, daß innerhalb eines Madenalters von 0 bis knapp 2 Tagen (1. Versuchsgruppe) gesicherte Gewichtsunterschiede der sich entwickelnden Königinnen weitgehend fehlen. Die Gewichtsunterschiede sind zufällig und waren mit 3 Ausnahmefällen, in denen eben geschlüpfte mit knapp 2-tägigen Larven verglichen wurden, mit dem klassischen $t$-Test nicht zu sichern.

Die Ausnahmefälle wiesen zweimal auf einen Gewichtsvorteil der Königinnen aus jüngeren (Zucht-Nr. 18 u. 19) und einmal auf einen solchen aus älteren Larven hin (Zucht-Nr. 1). In allen 3 Fällen handelt es sich um spätere Zuchtserien aus wiederholten Pflegefolgen. Bezeichnenderweise enthält das Protokoll der Zucht Nr. 18 den Hinweis, daß alle umgelarvten Maden auffällig groß waren. Auf der anderen Seite ist zu bemerken, daß die Vorgeschichte des Pflegevolkes von Zucht-Nr. 1 insofern von dem üblichen Gang der Pflege abwich, als es kurz nacheinander 8 mal zum Anpflegen von Larven für nur einen oder höchstens 2 Tage verwendet wurde. Es steht offen, ob das den Zuchtversuch beein fluß hat.

Wenn man mit Hilfe des parameterfreien Rangzahlentests von ManN und Whitney alle vergleichbaren Zuchten dieser Versuchsgruppe zusammenfaßt, ergibt der Gewichtsvergleich von Königinnen aus 0-bis 1-stündigen mit knapp 1-tägigen Maden (Zucht-Nr. 12, 13, 16, 17, 18, 20 u. 21), eine Irrtumswahrscheinlichkeit von $p=0,049\left(\mathbf{n}_{1}=75 ; \mathbf{n}_{2}=72 ; u=1,96\right)$, also eine nur recht schwache Sicherung. Ein Unterschied zwischen den Gewichten der Tiere aus 1-und knapp 2-tägigen Maden der Zuchten Nr. 2-18, 20 u. 21 ließ sich bei $p=0,142\left(n_{1}=195 ; n_{2}=260 ; u=1,47\right)$ statistisch überhaupt nicht nachweisen. Dagegen ergab sich bei Zusammenfassung aller Versuche, in 
denen Königinnen aus eben geschlüpften und knapp 2 Tage alten Maden verglichen wurden (Zucht-Nr. 1, 12, 13, 16-21), mit $\mathbf{p}=0,003\left(\mathrm{n}_{2}=101\right.$; $\mathbf{n}_{2}=108 ; \mathbf{u}=3,04$ ), eine gute Sicherung. Der durchschnittliche Gewichtsunterschied zwischen diesen beiden Königinnengruppen aller 9 Vergleichszuchten betrug jedoch nur $9,37 \mathrm{mg}$ bei einem mittleren Puppengewicht sämtlicher beteiligter Königinnen von $255,58 \mathrm{mg}$.

Deutlicher als in der 1. Versuchsgruppe war der Gewichtsunterschied der Königinnen in der 2. Versuchsgruppe, in der über 2-tägige Maden mit jüngerem Larvenmaterial verglichen wurden. Schon wenig über 2 Tage alte Maden ergaben in der Regel leichtere Königinnen. Die Unterschiede ließen sich meist schon in den Einzelzuchten mit dem t-Test statistisch absichern. Allerdings kamen auch hier Ausnahmen von der Regel vor, wie etwa in ZuchtNr. 22 (bei einer ähnlichen Vergangenheit des Pflegevolkes wie in Zucht-Nr. 1).

Ablauf und Gesamtergebnis der Versuche lassen keinen altersbedingten Unterschied im Fütterungsverhalten der $\mathbf{P}$ flegebienen gegenüber verschieden alten Zuchtlarven etkennen. Die bei der Aufzucht aus verschieden alten Maden erreichten Königinnengewichte waren unabhängig vom Alter der p flegenden Ammen.

\section{III. - UMLARVALTER UND RESTFUTTERMENGE}

Bei gleichzeitiger Verwendung verschiedener Larvenalter im gleichen Pflegevolk fiel auf, daß die Zellen der Königinnen, die aus 0 -tägigen Larven hervorgingen, größer, insbesondere länger waren, als die von Königinnen aus 1-oder 2-tägigen Maden (Abb. 1). Dieser Längenunterschied hat etwas mit der nach der Verpuppung in den Zellen zurückbleibenden unverbrauchten Restfuttermengen zu tun. Sie ist erwartungsgemäß von Zelle zu Zelle, von Volk zu Volk und von Pflegefolge zu Pflegefolge Schwankungen unterworfen. Darüber hinaus bleiben beim Umlarven jüngerer Maden größere Restfuttermengen in den Zellen zurück als bei Verwendung älterer Tiere. Tabelle 2 zeigt am Beispiel dreier Pflegefolgen, wie sich jeder Tag, den eine Made früher in königliche Pflege kommt, deutlich in einer Steigerung der Futtersaftrestmenge auswirkt. Die Gewichtsunterschiede lassen sich besonders in den ersten Pflegefolgen statistisch gut sichern (Spalte 8). Sie verlieren sich in den späteren Zuchtserien, wenn die Futtersaftmengen in den Zellen abnehmen (7. - 10 . Zuchtserie der Pflegefolge A 72).

In der Pflegefolge A (72) lassen sich auch Zusammenhänge zwischen den Futtersaftrestgewichten (Spalte 7) und der vom Volk angenommenen Zellenzahl erkennen. Zum Beispiel steigen die Restfuttermengen in der 8. Zuchtserie gegenüber der Zuchtserie zuvor bei geringerem Zellenansatz deutlich an. Im 


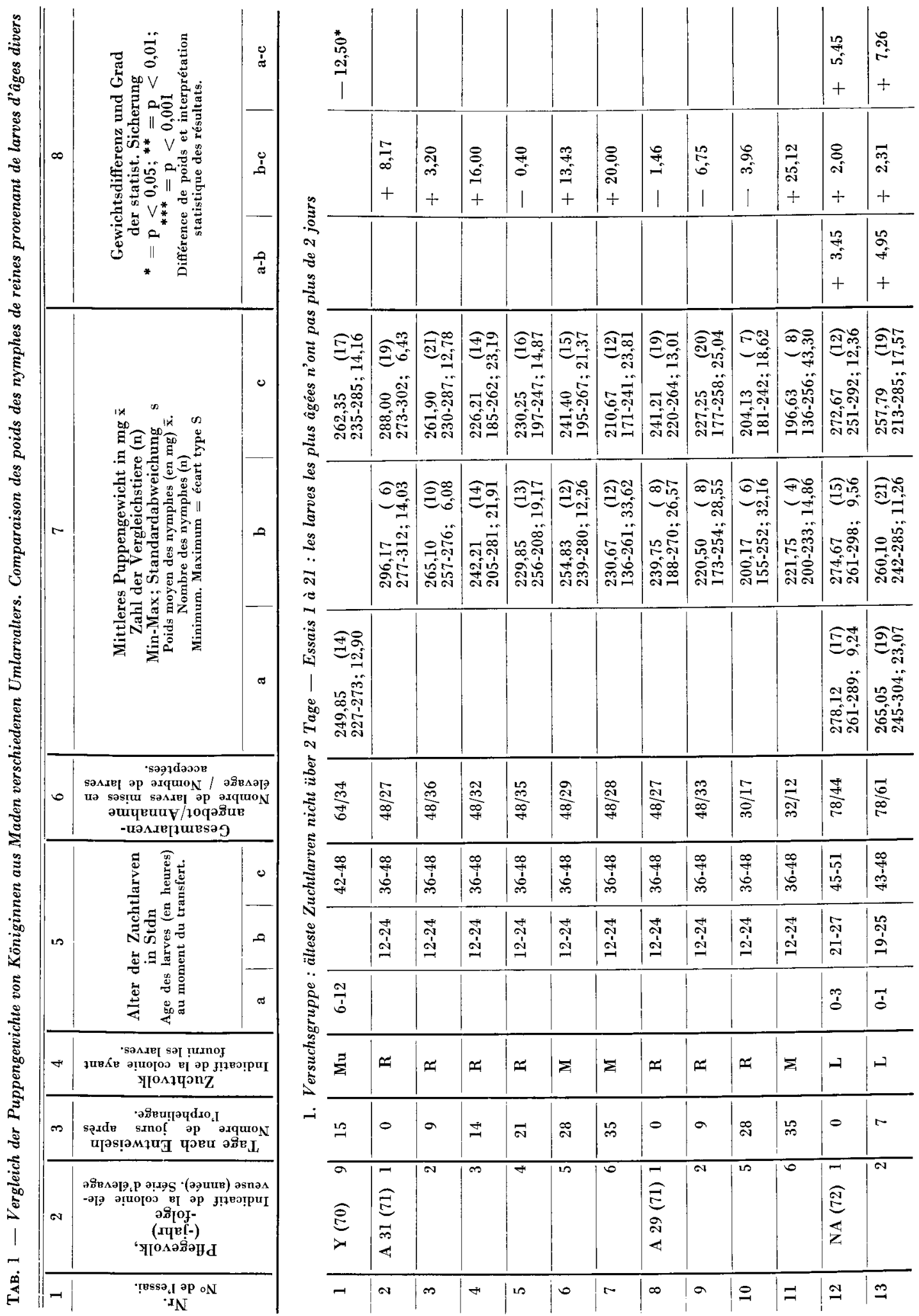



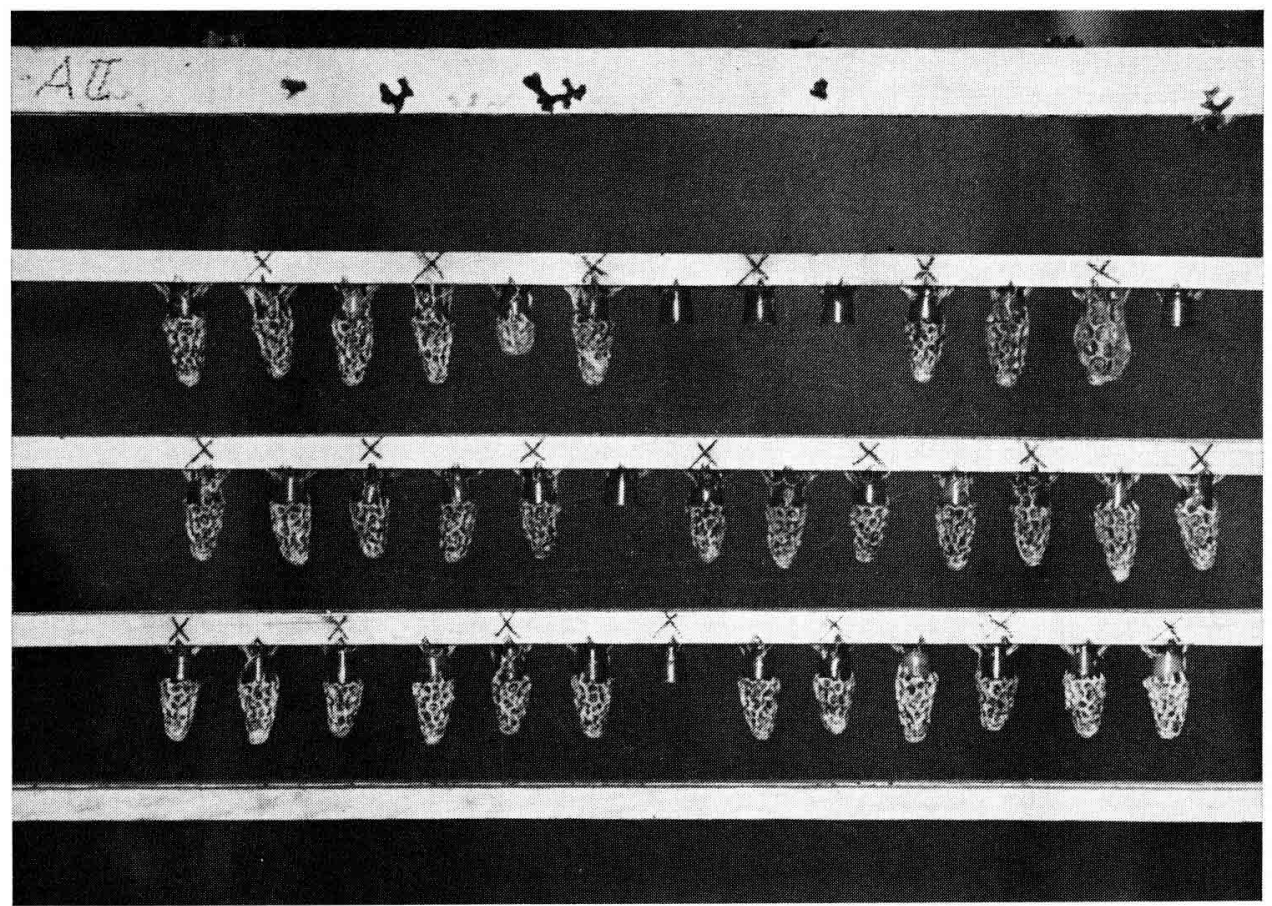

Авв. 1. - Zuchtrahmen mil Zellen über 1 - und 2-tägig umgelarvten Maden aus der Pflegefolge A (72), 2. Zuchtserie. Die Zellen über den 2-tägigen Maden sind auf den Zuchtlatten mit $x$ gekennzeichnet.

FIG. 1. - Cadre d'élevage avec cellules contenant des larves de 1 jour et 2 jours au moment du transfert. Elevage A (72), $2^{c}$ série. Les cellules contenant des larves de 2 jours sont marquées d'une croix.

übrigen ist eine kontinuierliche Abnahme der Restfuttergewichte vom Start der Zuchtversuche an festzustellen.

Der Unterschied in den durchschnittlichen Restfuttermengen bezogen auf das Larvenalter erscheint in einem besonderen Licht, wenn man die Erstversorgung der umgebetteten Maden nach einer bestimmten Anpflegezeit untersucht. Dabei stellt sich heraus, daß jüngere Maden aus der gleichen Zuchtserie in einer bestimmten Zeiteinheit weniger Futter bekommen als ältere Maden. Tabelle 3 vergleicht die Futtersaftversorgung von eben geschlüpften mit 1 und 2 Tage alten Arbeitermaden 24 Stunden nach dem Umlarven im gleichen Pflegevolk. Der Unterschied in den durchschnittlichen Futtersaftmengen, welche sich in dieser Zeit entsprechend dem Umlarvalter in den Zellen angesammelt haben, ist schon bei den wenigen Meßwerten statistisch hoch bis gut gesichert (Spalte 5). Wenn wir die Anfangsversorgung der verschieden alten Maden mit den Futtersaftrestmengen vergleichen, welche nach der Verpuppung in den Zellen zurückbleiden (Tabelle 2), kehren sich die Mengenverhältnisse gerade um (s. Diskussion). 


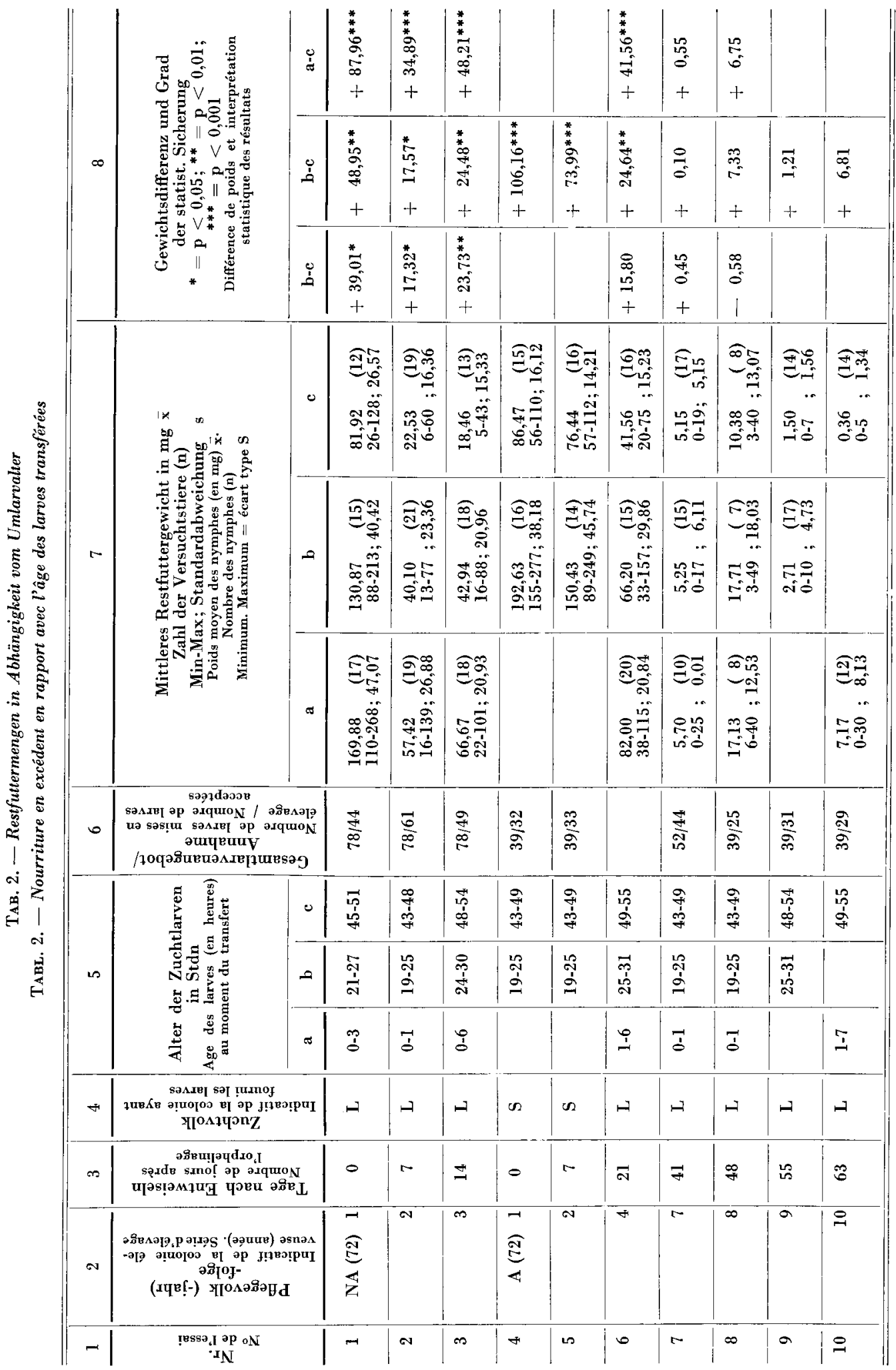


TAB. 3. - Nach 24 Stunden Anbrütezeit abgelagerte Futtersaftmengen bei Umbettung 1,2 und 3 Tage alter Maden

TABL. 3. - Quantité de gelée royale déposée après 24 heures de prise en élevage auprès de larves de 1,2 et 3 jours au moment du transfert.

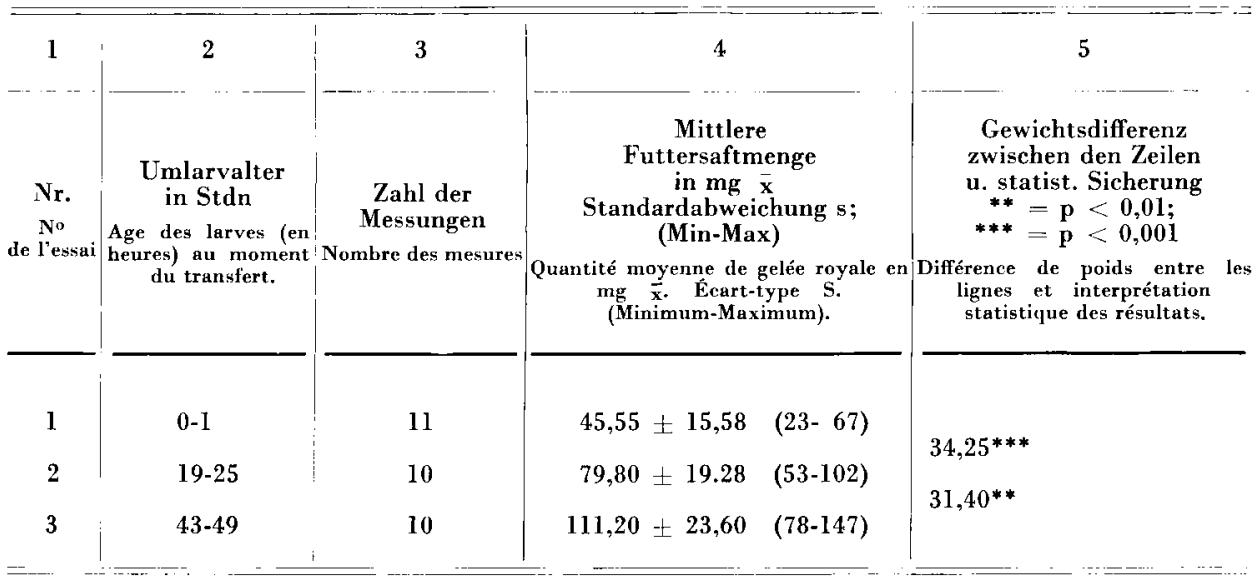

\section{IV. - RESTFUTTERMENGEN UND KONIGINNENGEWICHT}

Der in den Zellen zurückbleibende individuell und von Zuchtserie zu Zuchtserie schwankende Futtersaftrest gibt zur Frage Anlaß, ob eine Abhängigkeit zwischen der Überschußmenge und dem Gewicht der einzelnen Königinnen besteht.

Wenn wir diese Frage im Rahmen unserer Vergleichszuchten mit verschieden alten Zuchtmaden prüfen wollen, müssen wir jedes Larvenalter für sich betrachten. Das ist bei den aus Tabelle 2 in Tabelle 4 übernommenen Zuchtbeispielen geschehen. (Übersichtshalber sind hier in Spalte 4 u. 5 noch einmal die Mittelwerte der Puppen-und Futtersaftrestgewichte angegeben.) Wenn man die einzelnen Puppengewichte der Königinnen den in ihren Zellen zurückbleibenden Restfuttergewichten gegenüberstellt, errechnen sich die in Spalte 6 wiedergegebenen Korrelationswerte. Die Korrelationskoeffizienten sind mit ihrem teils positiven, teils negativen Vorzeichen willkürlich. Nur in einem von 26 Fällen besteht eine geringe Sicherung im Sinne einer positiven Abhängigkeit. Diese unerklärte Ausnahme ändert nichts am Gesamtergebnis der Untersuchung, das besagt : Restfuttermengen und Königinnenpuppengewichte stehen in keiner Beziehung zueinander. Zellen mit großen Futtersaftrestmengen können kleine, solche mit geringen Futtersaftresten große Königinnen beherbergen. Dies gilt nicht nur zu Beginn der Zuchtfolgen, wenn genügend Futtersaftüberschüsse vorhanden sind, sondern auch bei schwindenden Futtersaftresten in späteren Zuchtserien. 


\begin{tabular}{|c|c|c|c|c|c|c|c|c|c|c|c|c|c|}
\hline & & 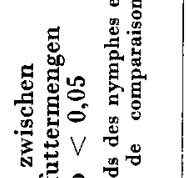 & 0 & $\begin{array}{l}\underset{\Xi}{\Xi} \\
0 \\
0 \\
+ \\
+\end{array}$ & $\begin{array}{l}\widehat{\sigma} \\
\stackrel{8}{8} \\
\stackrel{0}{0} \\
1\end{array}$ & $\begin{array}{l}\underset{3}{\Xi} \\
\tilde{\omega} \\
0 \\
+\end{array}$ & $\begin{array}{l}\underset{S}{\varrho} \\
\stackrel{0}{0} \\
+ \\
+\end{array}$ & $\begin{array}{l}\frac{0}{9} \\
\frac{9}{0} \\
+\end{array}$ & $\begin{array}{l}0 \\
0 \\
2 \\
0 \\
0 \\
1\end{array}$ & \begin{tabular}{l}
$\underset{E}{E}$ \\
\multirow{J}{+}{} \\
0 \\
+ \\
+
\end{tabular} & \begin{tabular}{l}
$\infty$ \\
\hdashline \\
0 \\
0 \\
0 \\
+
\end{tabular} & $\begin{array}{l}\underset{\mathbb{E}}{d} \\
g \\
0 \\
0 \\
+\end{array}$ & 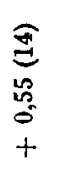 \\
\hline & 6 & 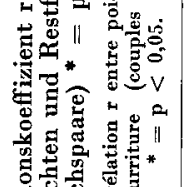 & $a$ & 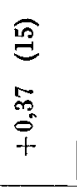 & $\begin{array}{l}\widehat{\text { త্ }} \\
\text { స̃ } \\
\text { ô }\end{array}$ & $\begin{array}{l}\stackrel{\infty}{=} \\
8 \\
8 \\
0\end{array}$ & $\begin{array}{l}\stackrel{6}{\varrho} \\
\stackrel{0}{0} \\
\stackrel{0}{0}\end{array}$ & 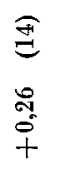 & $\begin{array}{l}\widehat{1} \\
= \\
\vdots \\
0 \\
+\end{array}$ & 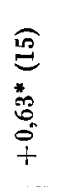 & $\begin{array}{l}\tilde{E} \\
⿱ 乛 \\
0 \\
+\end{array}$ & 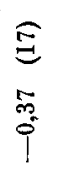 & \\
\hline & & 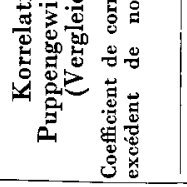 & $\approx$ & 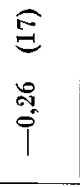 & 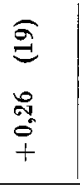 & 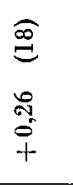 & & & 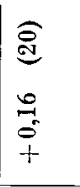 & 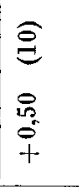 & $\begin{array}{l}\infty \\
-1 \\
0 \\
0\end{array}$ & & $\begin{array}{l}\stackrel{\Im}{\Xi} \\
\dot{\Xi} \\
i \\
0\end{array}$ \\
\hline 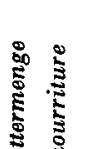 & & 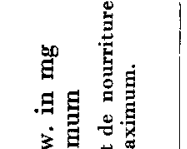 & 0 & 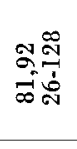 & $\begin{array}{l}\text { מ马: } \\
\text { ฟู่ }\end{array}$ & 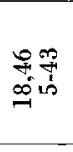 & 我胥 & 㨁: & 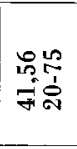 & 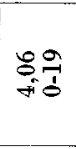 & 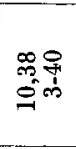 & 藏 & 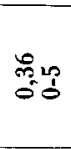 \\
\hline 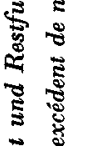 & 10 & 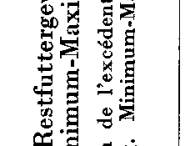 & $\therefore$ & 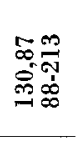 & 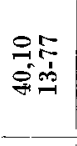 & 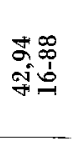 & 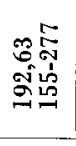 & 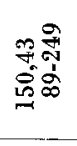 & 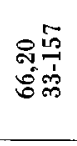 & $\begin{array}{l}\text { त̂ } \\
\text { ño }\end{array}$ & $\begin{array}{l}\text { E⿸广巳 } \\
\text { 亲的 }\end{array}$ & $\begin{array}{l}\text { E⿵ } \\
\text { id }\end{array}$ & \\
\hline 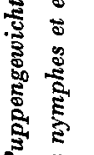 & & 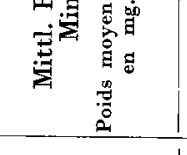 & $\pi$ & 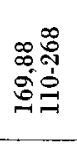 & 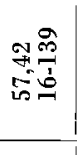 & 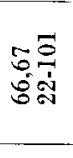 & & & 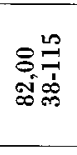 & $\begin{array}{l}\text { 붕 } \\
\text { no }\end{array}$ & $\begin{array}{l}20 \\
\stackrel{9}{=} \\
=1\end{array}$ & & 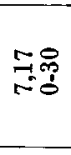 \\
\hline 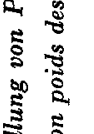 & & 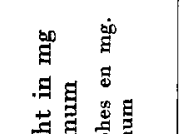 & 0 & 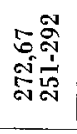 & 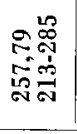 & 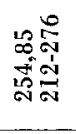 & 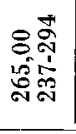 & 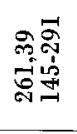 & 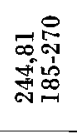 & 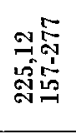 & 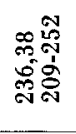 & 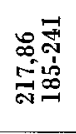 & 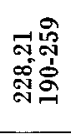 \\
\hline 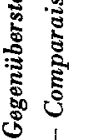 & + & 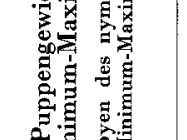 & $a$ & 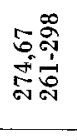 & 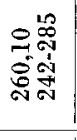 & 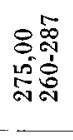 & 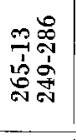 & 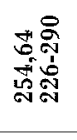 & 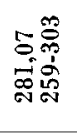 & 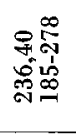 & 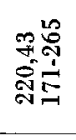 & 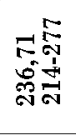 & \\
\hline l & & 这 & $\pi$ & 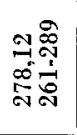 & 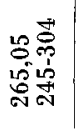 & 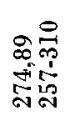 & & & 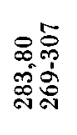 & 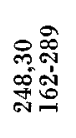 & 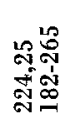 & & 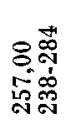 \\
\hline & & 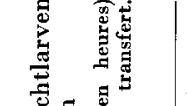 & 0 & $\begin{array}{l}\vec{n} \\
\text { in } \\
\text { ro }\end{array}$ & 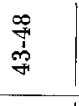 & \begin{tabular}{l}
$\vec{b}$ \\
0 \\
0 \\
\multirow{3}{*}{}
\end{tabular} & 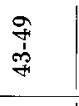 & $\begin{array}{l}\overrightarrow{1} \\
\stackrel{1}{1}\end{array}$ & 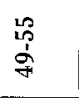 & 辛 & 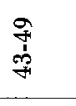 & $\begin{array}{l}\text { 落 } \\
\text { 呆 }\end{array}$ & $\begin{array}{l}\text { 帒 } \\
\text { ó }\end{array}$ \\
\hline & $m$ & 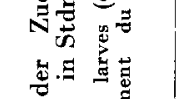 & $a$ & $\underset{\mathbb{N}}{\stackrel{N}{N}}$ & 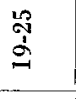 & 莡 & 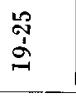 & 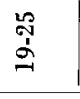 & $\begin{array}{l}\vec{p} \\
\text { i⿱宀 }\end{array}$ & $\begin{array}{l}\text { 岀 } \\
\text { فे }\end{array}$ & $\begin{array}{l}\text { a } \\
\text { a } \\
2\end{array}$ & 营 & \\
\hline & & 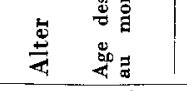 & $\approx$ & d & $\overrightarrow{8}$ & $\stackrel{i}{b}$ & & & $\stackrel{\varphi}{i}$ & $\bar{\delta}$ & $\vec{d}$ & & $\stackrel{I}{I}$ \\
\hline & $\infty$ & 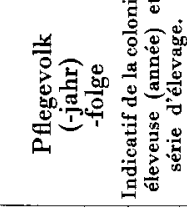 & & 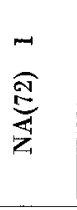 & $\boldsymbol{N}$ & m & 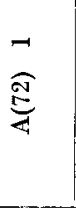 & N & 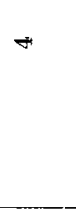 & $r$ & $\infty$ & $a$ & 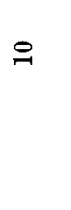 \\
\hline & -1 & 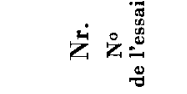 & & - & N & $\infty$ & $\overrightarrow{7}$ & is & 0 & $r$ & $\infty$ & $a$ & 윽 \\
\hline
\end{tabular}


Die individuelle Unabhängigkeit von Puppengewicht und Futtersaftrest ändert nichts an der Tatsache, daß beide Maßzahlen im Laufe der aufeinanderfolgenden Zuchten abnehmen. Ich habe diese Verhältnisse am Beispiel zweier lückenloser Zuchtfolgen in Abbildung 2 graphisch dargestellt. Diese Versuche, welche 1968 in Erlangen durchgeführt wurden, hatten den Zweck, das maximale Alter der Ammen zur Königinnenpflege zu ermitteln (WeIss 1972). In den beiden Pflegevölkern (Carnica-Rasse) war gleichzeitig mit der Königin auch sämtliche offene und gedeckelte Brut entnommen worden. Der verwendete Zuchtstoff gehörte in einem Fall der Carnica-, im anderen der Mellifica-Rasse an. Umgelarvt wurden stets gleichaltrige 3/4- bis 1-tägige Maden. Die Völker erhielten in 5-, später in 6-tägigem Abstand jeweils 24 Maden zur Pflege. Man sieht, daß die Futtersaftrestmengen bei gleichem, begrenztem Larvenangebot stetig und sehr schnell abnehmen, während die Gewichtskurven der Puppen nur zögernd folgen. Das Durchschnittsgewicht der Carnica-Königinnen steigt sogar in der 4. Zuchtserie nocheinmal kräftig an, während in der Mellifica-Zucht der Gewichtsabfall bei durchwegs höheren Annahmezahlen schon früher augenfällig wird. Ursache hierfür ist natürlich nicht die Rasse des Zuchtstoffes, sondern das individuelle Verhalten der P flegevölker.

Ich habe auch bei diesen beiden Zuchten nach einer möglichen Korrelation zwischen Puppengewicht und dem jeweils übrig gelassenen Futtersaftrest gesucht. Die Korrelationskoeffizienten (r) betragen in der Reihenfolge der einzelnen Zuchtserien im Falle der Carnica-Zucht : $-0,12,-0,55^{*},+0,33$, $+0,37,+0,22,-0,62^{* *},+0,34,-0,07,+0,46,-0,26,-0,04,+0,09$, $+0,10$ und in der Mellifica-Zuchtfolge : $-0,14,-0,47^{*},+0,53^{*},+0,24$, $+0,31,+0,39$. Es gibt in insgesamt 19 Fällen drei “ gerade noch 》 $(\mathbf{p}<0,05)$ und eine " gute 》 Sicherung $(p<0,01)$ der Abhängigkeiten. Die Sicherung betrifft aber sowohl positive als auch negative Korrelationswerte, was besagt, daß hohes Puppengewicht einmal mit großer und das andere Mal mit kleiner Futtersaftrestmenge gekoppelt war. Die Protokolle des Versuchsverlaufes sind nicht in der Lage, diesen Widerspruch zu klären. Von diesen Ausnahmen abgesehen, wird das allgemeine Resultat der in Tabelle 4 abgehandelten Versuche bestätigt : Zwischen den Puppengewichten und den Restfuttersaftmengen besteht keine gesetzmäßige Abhängigkeit.

\section{DISKUSSION}

a) Zwischen Königinnen aus eben geschlüpften, knapp 1-und knapp 2-tägigen Maden besteht ein geringer, mit dem Larvenalter ansteigender Gewichtsunterschied. Er war innerhalb der Einzelzuchten mit dem klassischen Testverfahren nicht sicher nachzuweisen, ließ sich aber bei Zusammenfassung 


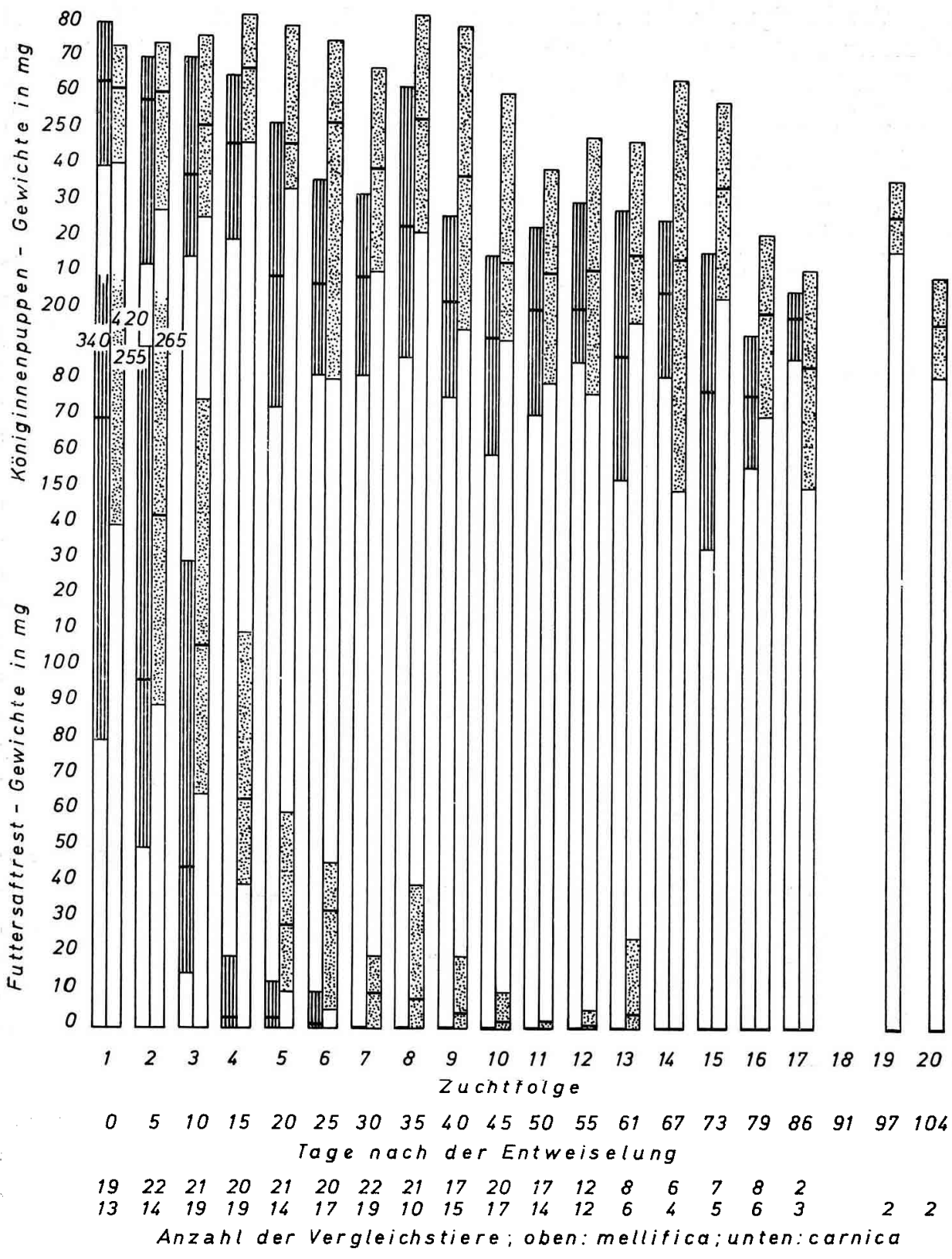

Авв. 2. - Durchschnittsgewichte mit Variationsbreiten der Königinnenpuppen und der zugehörigen Futtersaftreste aus zwei Sukzessivzuchten. Senkrecht schraffiert: Carnica-Pflegevolk mit Mellifica-Zuchtstoff; punktiert : Carnica- Pflegevolk mit Carnica- Zuchtstoff.

FIG. 2. - Poids moyen et espace de variation des nymphes de reines et des quantitês de nourriture en excédent correspondantes dans deux élevages successifs (en ordonnées-mg).

Hachures verticales : colonie éleveuse carnica et larves mellifica.

Pointillé : colonie éleveuse carnica et larves carnica.

Abscisses : No de la série d'élevage.

Nombre de jours après l'orphelinage.

Nombre des individus : en haut mellifica en bas carnica. 
aller einschlägigen Zuchten mit dem parameterfreien Test von MANN und Whitney wenigstens teilweise sichern. Erst beim Vergleich von Königinnen aus über 2 Tage alten Maden mit solchen aus jüngeren Larvenstadien kam es auch in den einzelnen Zuchten zu einem gesicherten Gewichtsunterschied.

Die immer wieder auftretenden indifferenten Fälle und Ausnahmen von der allgemeinen Tendenz scheinen etwas mit der Larvengröße zu tun zu haben. In manchen Völkern und zu manchen Zeiten wachsen Arbeiterlarven (auch Königinnenlarven) schneller oder langsamer als erwartet. Die Gründe hierfür mögen verschieden sein. Als ich bei kaltem Wetter ein Volk beheizte, um die Königin zu besserer Legetätigkeit anzureizen, entstanden für ihr Alter auffällig große Larven, was auf eine Temperaturabhängigkeit der Larvenentwicklung hindeutet. Als ich dagegen ein etwas stechlustiges Volk, von dem ich gelegentlich Zuchtstoff entnahm, vor dem Absperren der Königin auf kurze Entfernung verstellte, um die Flugbienen zu reduzieren, protokollierte ich später relativ kleine altersdatierte Larven. Wenn in diesem Fall nicht auch die Temperatur einen Ein fluß hatte, war möglicherweise die gestörte Alterszusammensetzung der Bienen an dem geringeren Wachstum der Larven schuld. Ein anderes Mal blieb diese Wirkung aus. Völker können offenbar in der gleichen Situation verschieden reagieren. Während das eine die zu groß gewordenen Larvenbezirke vielleicht nur ungenügend versorgte, könnte das andere die überzähligen Larven ausgeräumt und die verbliebene Brut umso besser gep flegt haben. Letztlich ist die Larvenpflege wohl auch erblichen Einflüssen unterworfen, so wie auch die Entwicklungszeit der Eier und die Wachstumsgeschwindigkeit der Larven genetisch beeinflußt sein können.

Beträchtliche Größenunterschiede lassen sich aber auch häufig bei altersgleichen Einzellarven gleicher Abstammung und Aufzucht feststellen. Zurückgebliebene Tiere gibt es, solange die Larven noch in den Arbeiterzellen liegen, wie auch bei ihrer königlichen Weiterp flege. Hier mögen Zufälligkeiten in der Pflege oder besondere Temperaturein flüsse im Spiele sein. Larven am Rande des Brutnestes oder des Zuchtrahmens fallen besonders gerne aus dem allgemeinen Rahmen.

b) In einem früheren Untersuchungsbefund über die Abhängigkeit von Königinnenausbildung und Madenalter (WEIB 1971) erzeugte ein alterndes Pflegevolk aus 2 Tage alten Maden statistisch « eben noch ) gesichert größere Königinnen als aus 1-tägigen Tieren, während es zu Beginn der Zucht gerade umgekehrt reagierte. Das jetzt vorliegende größere Untersuchungsmaterial brachte keine Bestätigung dieser Beobachtung. Die Vermutung, daß überalterte Pflegebienen ältere bzw. größere Maden angeborenermaßen besser versorgen könnten als jüngere, trifft also nicht zu. Der frühere Untersuchungsbefund ist eine Ausnahme vom allgemeinen Verhalten.

Solche Ausnahmen sind keine U̇berraschung. Sie treten bei verschiedensten 
Aufzuchtfragen auf und sind oft auch bei eifrigster Durchforstung der Versuchsprotokolle nicht stichhaltig erkläbar. Man ist in solchen Fällen geneigt, an das zufällige Auftreten einseitiger Fütterungsspezialisten im Pflegevolk oder einfach an “ Launen » desselben zu glauben.

c) Die Versuche haben gezeigt, daß sich aus über 2 Tage alten Arbeitermaden in der Regel statistisch gesichert leichtere Königinnen entwickeln als aus jüngeren Tieren. Man könnte als erstes vermuten, daß daran einfach der größere Nährstoffgehalt des Königinnenfutters schuld sei, welches die Maden entsprechend dem Zeitpunkt ihrer Umbettung früher oder später erhalten. Damit lassen sich aber schwerlich die Untersuchungsbefunde von STABE (1930) und WANG (1965) vereinbaren, wonach die Arbeitermaden in den ersten 3 Larventagen rascher als Königinnenmaden wachsen (Abb. 3). Letztere holen erst am Ende des 4. Larventages an Gewicht auf, um die Arbeitermaden schließlich zu über flügeln. Das heißt also : Je später die Arbeiterlarve umgebettet wird, desto größer ist sie gegenüber einer gleichaltrigen Königinnenlarve. Sie ist auch größer als eine gleichaltrige, aber schon früher umgebettete Arbeitermade, da diese mit Einsetzen der königlichen Fütterung nur verlangsamt weiterwächst.

Nun beginnt für die älter umgebettete, (größere) Arbeiterlarve die Phase der beschleunigten Königinnenentwicklung früher als für eine jünger umgebettete. Man möchte deshalb erwarten, daß sich die ältere Arbeitermade mit dem Wachstumsvorsprung gegenüber einer gleichaltrigen, früher umgebetteten und in ihrer Größenentwicklung zurückgehaltenen Larve zu einer größeren Königin entwickeln würde. Gerade das Gegenteil ist aber der Fall. Die ältere Arbeitermade ergibt eine kleinere Königin und umgekehrt. Das bedeutet, daß schon während der ersten Larvenzeit etwas geschieht, was die spätere Gewichtsentwicklung der heranwachsenden Königinnen beeinflußt.

Als Erklärung könnte man an einen praedeterminierenden Wachstumsfaktor im Königinnenfuttersaft der jungen Maden denken, wie man anderseits auch einen Faktor mit umgekehrter Wirkung im Arbeiterfuttersaft annehmen kann. Während die frühen Wachstumsunterschiede durch einfache Ernährungseinflüsse hervorgerufen würden, könnte die spezifische Wachstumsintensität der älteren Larve auf hormonalen Vorgängen beruhen, welche durch den praedeterminierenden Faktor im jungen Larvenstadium ausgelöst wurden.

Eine weniger aufwendige Erklärung gründet sich auf die berechtigte Ansicht, daß das stärkere Wachstum der jungen Arbeiterlarven gegenüber gleichaltrigen Königinnenlarven nur durch einen höheren Wassergehalt der ersteren vorgetäuscht ist. Jüngere Arbeiterlarven enthalten nach Strauss (1911) mehr Wasser als ältere. Vor allem sind sie nach MeLampy u. M. (1940) auch wasserreicher als gleichaltrige Königinnenlarven. Dazu passend stellte 


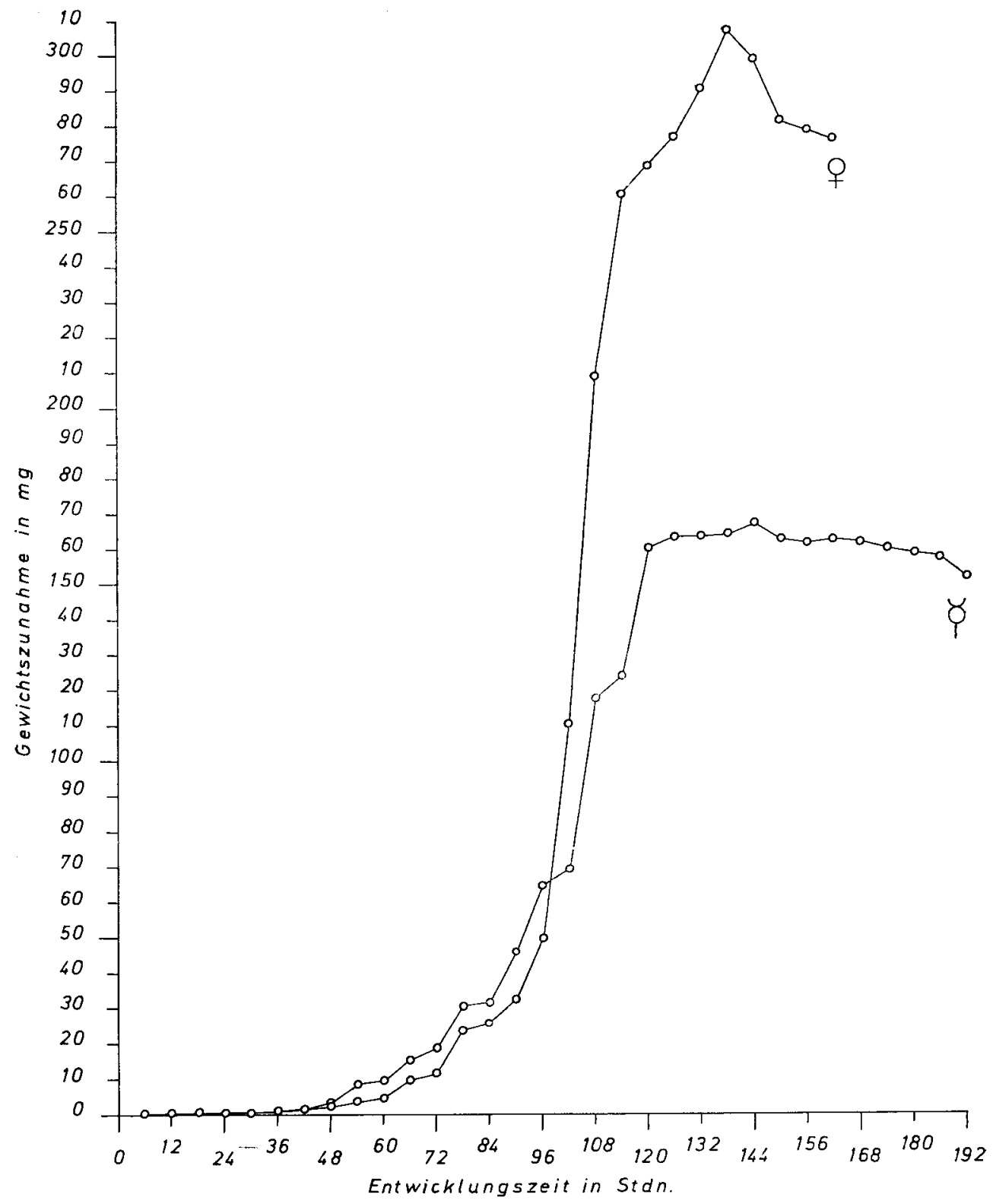

Aвв. 3. - Wachstumskurven von Königinnen-und Arbeiterinnenlarven, gezeichnet nach den Wägeergebnissen von Wang (1965).

FIg. 3. - Courbes de croissance des larves de reine et d'ouvrières. D'après les données de Wang (1965). Abscisses: Temps de développement (en heures).

Ordonnées : gain de poids (en $\mathrm{mg}$ ). 
Wirtz (1973) in histologischen Beobachtungen fest, daß im Fettkörper junger Arbeiterlarven große Flüssigkeitsvacuolen auftreten, die bei Königinnenlarven fehlen. Strauss (1911) fand außerdem in den ersten 3 Entwicklungstagen der Arbeiterinnenlarve wenig Glykogen und Fett vor, was sich auf späterem Altersstadium änderte. Nach 3 Tagen Larvenzeit enthielten Königinnenlarven nach Melampy u. M. (1940) mehr Fett als gleichaltrige Arbeiterlarven. Mit Ausnahme des Wassergehaltes, der bei 3-tägigen, im Labor mit Königinnenfuttersaft aufgezogenen Larven etwas höher lag als bei Tieren, die mit Arbeiterfuttersaft ernährt wurden, bestätigen auch Dixon u. Shuel (1963) einen deutlich höheren Lipid-und Glykogenanteil der königlich ernährten Tiere. Höher war außerdem der Anteil an nicht glykogenartigen Kohlenhydraten, während der Stickstoffanteil etwas niedriger lag. Die Arbeiterlarven machen am Ende ihrer Ernährungsperiode mit Futtersaft gegenüber gleichaltrigen Königinnenmaden einen unterernährten Eindruck. Es ist bezeichnend, daß sie sich bei künstlicher Weiterernährung mit Arbeiterfuttersaft im Labor nicht verpuppen, was sie bei Verabreichung von Mischfutter aber tun (v. RHEIN 1933). Shuel u. Dixon (1968) erreichten dasselbe durch Hinzufügung von Zucker zum Arbeiterfuttersaft. Die mangelhafte Ausrüstung der Arbeiterlarve mit Reservestoffen könnte letztlich auch dafür maßgebend sein, daß in der künstlichen Nachschaffungszucht die älter umgebettete Arbeitermade ein geringeres Adultgewicht erreicht als jünger umgebettete Tiere, obwohl letztere auf gleichem Altersstadium weniger wiegen als das später umgelarvte Tier.

Eine dritte Erklärungsmöglichkeit stützt sich auf Beobachtungen anläßlich jüngster vergleichender Versuche über die Zuchtpraktiken des “ einfachen » und « doppelten » Umlarvens (WeIss, in Vorber.). Dabei hat sich gezeigt, daß jüngere Larven eine mögliche kurze Hungerperiode nach dem Umlarven ohne jegliche Beeinträchtigung überstehen, während älter umgelarvte Tiere bei ihrem größeren Futterverbrauch Gewichtseinbußen erleiden können. Da bei den Versuchen zur Abhängigkeit des Adultgewichtes vom Madenalter stets " trocken » umgelarvt wurde, kann dies die Erklärung für den Wachstumsvorteil der jünger umgebetteten Maden sein.

d) Die königlichen Maden leben in einem optimalen Pflegevolk stets im Futterüberschuß. Das Futtersaftpolster, auf dem sie liegen, wächst mit ihrem Alter. Bezeichnenderweise sammelt sich in einer bestimmten Zeiteinheit in den Zellen neu umgebetteter Maden umso mehr Futtersaft an, je älter die Maden beim Umlarven sind. Zum Beispiel wog der Futterüberschuß 24 Stunden nach dem Umbetten 1-tägiger Larven fast doppelt soviel wie bei der Verwendung eben geschlüpfter Tiere. Man möchte annehmen, daß jedem Madenalter entsprechend der Pflegekraft der Ammenbienen ein bestimmter « Sollwert » an “ Überschußfutter » zusteht, den es so rasch als möglich zu erreichen gilt. Allerdings lassen die zunächst mit größeren Futtersaftüberschüssen ver- 
sorgten älteren Maden am Ende ihrer Entwicklung weniger Futter zuzück als ihre jünger umgebetteten Schwestern. Das dürfte mit der längeren königlichen Pflegedauer der letzteren zu erklären sein. Die jünger umgelarvten Maden gelangen schon früher in den Genuß des königlichen Futtersaftüberschusses, der sich mit jedem neuen Larventag weiter erhöht. Damit scheinen sich die Pflegebienen bei der Bemessung der Futterabgabe an die königlichen Larven zumindest nicht ausschließlich nach deren Alter bzw. Größe zu richten. Welches genaue Regulationsprinzip der Larvenversorgung zugrunde liegt, ist jedoch nicht bekannt.

e) Nach der Verpuppung der Königinnenmaden bleibt in einem optimalen P flegevolk ein Futtersaftrest in der Weiselzelle zurück. Er ist auch bei gleichen Umlarv-und Pflegebedingungen Schwankungen von Zelle zu Zelle unterworfen, die offenbar weniger mit dem unterschiedlichen Appetit der Maden als mit Zufälligkeiten in der Larvenversorgung durch die Pflegebienen zu tun haben. Gleiche Aufzuchtbedingungen vorausgesetzt, besteht keine Beziehung zwischen der Restfuttermenge und dem Königinnengewicht.

Dieser Sachverhalt trifft nicht mehr zu, wenn verschieden alte Larvenstadien zur Zucht verwendet werden. Da die Restfuttermengen verschiedener Umlarvalter echte Unterschiede aufweissen, ist in allen Fällen, wo das Königinnengewicht vom Umlarvalter beeinflußt wird, auch mit einer positiven Korrelation von Königinnengewicht und Futtersaftrestmenge zu rechnen. Das ist beim Vergleich von über 2-tägigen mit jüngeren Maden sicher der Fall. Die positive Beziehung Futtersaftrestmenge und Königinnengewicht ist dann allerdings nicht kausal, sondern kommt einfach dadurch zustande, daß beide Faktoren in gleicher Weise vom Umlarvalter abhängen.

Auch beim Vergleich verschiedener Zuchtserien derselben Zuchtfolge ist mit einer sekundären Abhängigkeit von Königinnengewicht und Futtersaftrestmenge zu rechnen. Die durchschnittlichen Futtersaftreste nehmen mit jeder neuen Serie ab, um schon nach wenigen Zuchten praktisch auf einem Nullwert zu landen. Erst dann scheinen auch die Königinnengewichte deutlicher zu fallen. Später mögliche Gewichtsanstiege hängen in der Regel mit reduzierten Annahmezahlen zusammen. Welche Ursachen bei den Schwankungen der durchschnittlichen Königinnengewichte von Serie zu Serie noch beteiligt sein können, ist im Einzelnen noch ungeklärt.

f) Für die Praxis bestätigen die Versuche einmal mehr, daß ein Umlarvalter bis zu $11 / 2$ Tagen züchterisch voll vertretbar ist. Nachdem sich bereits früher gezeigt hat, daß innerhalb dieses Alters die gezüchteten Königinnen in ihren kastentypischen Merkmalen nicht zu beanstanden sind (Weiss 1971), darf man nunmehr auch die Gewichtsentwicklung dieser jungen Larvenstadien als optimal ansehen. Der schwer zu sichernde Gewichtsunterschied zwischen Königinnen, die aus verschieden alten bis knapp 2-tägigen Arbeiterlarven 
hervorgehen, ist so gering, daß er in der praktischen Zuchtarbeit überhaupt keine Rolle spielt. Erst um den 2. Larventag herum scheint der kritische Zeitpunkt erreicht zu werden, wo mit einem bedeutsameren Gewichtsverlust zu rechnen ist.

Eingegangen im November 1973.

Reçu pour publication en novembre 1973.

\section{RÉSUME}

A fin d'élucider la question de savoir s'il existe un rapport entre le poids de la reine adulte et l'âge auquel a été tranférée la larve dont elle provient, de nouvelles expériences ont été faites avec des larves d'ouvrières d'âge variable mais de même origine et élevées dans des conditions identiques (même série d'élevage et même colonie éleveuse). Au lieu du poids de l'adulte, qui n'est habituellement pas déterminable avec précision, j'ai considéré le poids de la nymphe (WEIss, 1967).

$\mathrm{Si}$, à l'intérieur de chaque élevage on considère les différences de poids moyens entre des nymphes provenant de larves transférées à des âges divers mais au maximum à l'âge de deux jours, on constate une certaine irrégularité. Le test $t$ ne permet de mettre en évidence que rarement des différences statistiquement significative. (Tableau $1, n^{\circ} 1-21$ ). On a alors regroupé tous les élevages comparables et traité les données au moyen d'un test non paramétrique. La comparaison des reines provenant de larves âgées de 1 jour et de tout juste 2 jours (19 élevages et 455 reines) ne donne pas de différences statistiquement significatives. La comparaison des reines provenant de larves fraîchement écloses et de larves âgées d'à peine un jour (7 élevages et 147 reines) ne donne que des différences faiblement significatives. Par contre, la comparaison des reines provenant de larves fraîchement écloses et de larves âgées de tout juste 2 jours (9 élevages et 210 reines) montre que des différences de poids statistiquement significatives peuvent être plus aisément mises en évidence. Si l'on compare les poids des reines provenant de larves âgées d'un peu plus de 2 jours avec les poids des reines provenant de larves âgées d'à peine un jour ou fraîchement écloses on constate que les différences sont souvent hautement significatives et ceci déjà à l'intérieur d'un même série et à l'aide du test $\mathbf{t}$ (Tableau $1, \mathbf{n}^{0}$ 22-32).

Les larves relativement âgées au moment du transfert et principalement celles qui ont plus de 2 jours, donnent donc des reines qui sont plus petites que celles qui proviennent de larves fraîchement écloses ou âgées d'un jour. Ceci paraît en contradiction avec le fait que les larves transférées à un âge plus avancé bénéficient de la phase de croissance qu'elles ont eue comme larves d'ouvrières ( $\mathbb{W}_{A N G}, 1965$ ). Le fait qu'en cas de soins ultérieurs de même nature la larve d'ouvrière transférée plus jeune rattrape finalement en poids les individus transférés plus tard semble marquer l'action d'un « facteur de croissance prédéterminant " présent dans la gelée royale du premier âge larvaire. Ce phénomène peut toutefois être aussi bien en rapport avec le fait que la jeune larve d'ouvrière, dans les premiers jours de son développement, prend il est vrai davantage de poids, mais que ce gain de poids est davantage représenté par de l'eau que par des substances de réserve, la larve royale recevant une part plus importante de ces dernières. Cette “ sous-alimentation " au cours des premières heures de la vie larvaire se manifesterait par la suite comme un facteur d'inhibition de la croissance de la larve royale. Une troisième hypothèse s'appuie sur des expériences toutes récentes portant sur le double transfert comparé au transfert simple. Ces expériences ont montré que les très jeunes larves supportent sans inconvénient une courte période d'inanition aussitôt après le transfert « à sec » 
alors que les individus transférés à un âge plus avancé peuvent présenter une perte de poids préjudiciable (WEISs, en préparation).

Des expériences particulières ont montré que des larves qui sont transférées à différents âges reçoivent dans la même unité de temps des quantités excédentaires de gelée royale d'importance variable. Des larves transférées aussitôt après leur éclosion reposent après 24 heures sur une quantité de gelée royale qui est inférieure à celle sur laquelle reposent des larves transférées à un jour, laquelle est elle-même inférieure à celle qui correspond à des larves de 2 jours (Tableau 3). Après la nymphose, il reste toutefois dans les cellules occupées par les larves les plus jeunes, des quantités plus importantes de gelée royale inutilisée que dans les cellules occupées par des larves transférées à un jour. Les cellules occupées par les larves transférées à 2 jours en contiennent encore moins. (Tableau 2). Le temps passé par la larve dans la cellule royale détermine l'importance du reste de gelée inutilisée.

La quantité de gelée royale restant après la nymphose influence de façon très nette la longueur de la cellule. Les cellules royales de larves transférées jeunes sont, en général, plus longues que celles de leurs sœurs de même âge transférées plus tardivement, alors même que les poids des nymphes ne présentent pas de différence ( $F i g .1)$.

Il n'y a pas de corrélation entre le poids des nymphes de reines provenant de larves de même âge au moment du transfert et la quantité de gelée royale restant au fond des cellules.

Lorsqu'une même colonie éleveuse est utilisée de façon continue pour des élevages de reines on constate une diminution rapide des quantités de gelée royale en excédent. Après 4 ou 5 séries d'élevages successifs on ne trouve plus que des traces de nourriture au fond des cellules (Fig. 2). Dès ce moment le poids des nymphes commence à baisser de façon très nette alors que dans les premières séries il est variable. Par la suite on peut éventuellement constater des remontées de poids mais elles ne sont que partiellement en rapport avec une baisse du pourcentage des acceptations. Les causes de ces oscillations ne sont pas clairement établies.

Les recherches effectuées apportent la justification de l'emploi de la méthode du transfert des larves en apiculture pratique même si on se place au point de vue de l'obtention de reines d'un poids aussi élevé que possible. La tendance des jeunes larves à se développer en reines plus grosses est pratiquement sans signification tant qu'on reste dans la limite de 1 jour $1 / 2$ pour l'âge des larves à transférer. En utilisant les larves de 1 jour qui se laissent le mieux manipuler on devrait normalement se trouver dans des conditions optimales du point de vue biologique.

\section{LITERATUR}

Dixon S. E. u. Shuer R. W. : Studies in the mode of action of royal jelly in honeybee development. III. The effect of experimental variation in the diet on growth and metabolism of honeybee larvae. Can. J. Zool. 41, 733-739 (1963).

Jordan R. : Die Zucht der Königin ausgehend vom Ei. Bienenvater 81 (1) 3-7 (1960)

Krasnopojev (Pschelovodstvo 1949) nach Reininghaus, H. : Ei oder Made? Westf. Bz. 67 (6) 146-147 (1953).

LAwRECHIN F. A. : Eine vergleichende Gewichtscharakteristik von unfruchtbaren (?) Königinnen grauer kaukasischer Gebirgsbienen Apis mellifera caucasica Grob., die aus Eiern oder Larven gewonnen wurden. XIX. Int. Bz. Kongr. Prag 1963; Org. Ref. (dtsch. Fass.) II, S. 300-303.

Melampy R. M., Willis E. R. u. Mc Gregor S.E. : Biochemical aspects of the differentiation of the female honeybee (Apis mellifica L.) Physiol. Zool. 13, 283-293 (1940).

Niкоцau D. : Gute Weisel aus Schwarmzellen (rum.). Apicultura Bukarest 38, Aug.-H. S. 27 (1960) Ref. : Arch. Bienenkde. 38, 58 (1961). 
Rheiv W, von : Über die Entstehung des weiblichen Dimorphismus im Bienenstaate. Roux. Arch. Entw. Mech. d. Organ. 129, 601-655 (1933).

ShuEL R. W. u. Drxon S. E. : The importance of sugar for the pupation of worker honeybee. J. Apic. Res. 7, 109-112 (1968).

STABE H. A. : The rate of growth of worker, drone and queen larvae of the honeybee, Apis mellifera Linn. J. econ. Ent. 23, 4.47-453 (1930).

Strau B J. : Die chemische Zusammensetzung der Arbeitsbienen und Drohnen während ihrer verschiedenen Entwicklungsstadien. Z. Biol. 56, 347-397 (1911).

WaNg Der-J. : Growth rates of young queen and worker honeybee larvea. J. Apic. Res. 4. (1) 3-5 (1965).

Weaver N. : Effects of larval age on dimorphic differentiation of the female honey bee. Ann. Ent. Soc. Amer. 50 (3) 283-294 (1957).

WeIss K. : Zur vergleichenden Gewichtsbestimmung von Bienenköniginnen. Z. Bienenf. 9 (1) $1-21(1967)$.

: Über Ausbildung und Leistung von Königinnen aus Eiern und jungen Arbeitermaden. Apidologie 2 (1) 3-47 (1971).

- Verlauf und Beschaffenheit aufeinanderfolgender Zuchtserien im unverjüngten Pflegevolk. Apiacta VII (3) dtsch. Fass. 110-114 (1972).

- $\quad$ : Neue Untersuchungen zum « doppelten Umlarven ». Apidologie 5 (3) (1974).

WIRTz P. : Differentiation in the honeybee larva. Meded. Landbouwhogeschool Wageningen 73-5, 1-155 (1973).

Wolosiewitsch A. P. : Vergleichende Bewertung der Methoden der künstlichen Königinnenzucht. Ref. von H. Maul : Hess. Biene 90 (12) 352-353 (1954).

Woyke J. : Bedingungen der Aufzucht und die Zahl der Spermien, die die Samenblase der Königin erreichen. XXI. Int. Bz. Kongr. Maryland 1967, Org. Ref. (dtsch. Fass.) Nr.85. 